\title{
Research of Route Discovery Using Proficient Route Encounter Algorithm in MANET
}

\author{
Saritha Arumugam, N. Kumar
}

\begin{abstract}
The objective of the research work is proposed an efficient node to node route discovery in Mobile AD HOC network based on neighboring nodes resent route discovery. MANET is always depends on selected and constant path with extended time period subsequently, and the flexibility of the battery power will be condensed in searching end nodes which roots numerous path failure. In most of the scenario path failure origins consistent route discovery which is normally reduces the efficiency and it will increase the dependency in computational overhead of the nodes in the mobile routing environment.In order to overcome such failure in MANET it is proposed to implementing route discovery methodologiesand it will be done through neighboring nodes with its resent history log which is an efficient factor to reduce delay between nodes and improve consistency comparing with existing system. DSR were experimentally implemented and compared to find the end to end delay, throughput and packet delivery ratio between nodes. The proposed work is focused towards effective node discovery through Proficient Route Encounter (PRE) algorithm which minimize the delay and energy resource of the path finding node, time taken to reach its requested node and maximize packet delivery ratio and its energy efficiency.
\end{abstract}

Keywords--- PREA, EDA, DSR, MANET, NS-2.

\section{INTRODUCTION}

In the past few decade routing protocols for mobile ad hoc networks produce a lot of control activity when node versatility causes interface states and the system topology to change much of the time. Then again, assets, for example, data transmission and battery control are typically seriously obliged in such systems. In this manner, limiting the control movement to set up and keep up routing configuration is one of the fundamental difficulties in the structure of adaptable directing conventions for MANET. One way to deal with opposed to proactively [1]. Similarly a source node communicates a course disclosure control message when it needs to, and moderate hubs that get such message recommunicate it. A typical limitation to flooding is that a hub re-transmits a given course revelation ask for message just once and copies of a course ask for message are disregarded. This sort of flooding is utilized, for instance, in Ad Hoc on Dynamic source Routing protocol (DSR) are the most commonly used methodology [2]. Basically the hub in a MANET demonstrations both as a correspondence end and a switch. Dynamic source Routing protocol (DSR) is an on demand protocol, each node receive a route request packet

\footnotetext{
Manuscript received September 16, 2019.

Saritha Arumugam, Assistant Professor, Department of Computer Science and Engineering, Vels Institute of Science, Technology and Advanced Studies, Chennai, Tamil Nadu, India.(e-mail: saritha.se@velsuniv.ac.in)

N. Kumar, Associate Professor, Department of Computer Science and Engineering, Vels Institute of Science, Technology and Advanced Studies, Chennai, Tamil Nadu, India. (e-mail: kumar.se@ velsuniv.ac.in)
} limit control traffic is to build up routes on interest as

from its neighbor in the network, in DSR route is always discovered with high efficient and bandwidth consumption.

Since MANETs have constrained radio correspondence transmission capacity and use battery control, their conventions, including the directing conventions, must be productive in the utilization of data transfer capacity and vitality. A noteworthy method to ration data transfer capacity and vitality is lessening control overhead. There are two classes of impromptu directing conventions, the proactive and the on-request conventions. In the proactive steering conventions, a hub regularly keeps up a nearby directing table that has a passage for every goal in the system [3, 4]. Route discovery plays a significant role in various mobile ad hoc protocols which is based on flooding. Be that as it may, flooding experiences high overhead, which can build conflict and correspondence delays. The measure of communication age contrasts protocol (DSR) among hubs in MANET. Accordingly, there may be hubs that have much self-advantage in communication with nodes with as much other-advantage in correspondence $[5,6]$. The circumstance that the proportion of self-advantage correspondence to other-advantage correspondence contrasts among nodes isn't reasonable in light of the fact that oneself advantage correspondence is the benefit for the hub and other-advantage correspondence has no benefit for the node.

\section{RELATED WORKS}

In the prevailing work on simple route discovery has an execution part in such a way will rely upon the hubs versatility forms. In the standard mobility developments our recreations show that route discovery cost can be decreased by an order of magnitude, a significant gain given that route discovery is a major source of routing overhead in ad hoc networks in MANET environment [7]. An analytical framework for the characterization of link behavior in MANETs to precisely describe the time period of a wireless connection which is presented in [8] computed lifetime of a link concluded with two-state Markov model and that the systematic resolution tracks thoroughly, the results attained over and done with discrete event simulations. The benefit of this framework is that it exactly defines link dynamics as a purpose of node flexibility. The link stability predictionbased routing (LSPR) algorithm [9] uses comparativesignal and the distance flanked by various neighbor nodes to calculate the malicious link interval to forecast link immovability. The link stability improved routing for mobile ad hoc networks was proposed in MANET [10]. The preceding stability concerned with routing algorithms frequently focus in what way to determine a constant route,

Published By:

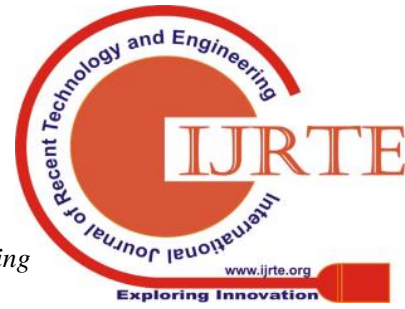




\section{RESEARCH OF ROUTE DISCOVERY USING PROFICIENT ROUTE ENCOUNTER ALGORITHM IN MANET}

but hardlydeliberate the flexibility to build route variation to monitor node's motion.

The link age ending time (LET), which is castoff to evaluate the permanency of link and it is intendedto measure accuracy in establishment with the discovery of some obtainable and constant routes in volatile methodology [11]. Accordingly in most of the proactive routing protocol ADOV and DSDV in MANET environment like energy channel frequency of a node is literally affected but not simply by an individual but also by its adjacent data streams as well $[12,13.14]$. The network environment reservations has connectivity by selecting a path conferring for the enduring battery life of nodes laterally with the route. The route might be carefully chosen with tiniest over-all broadcasting power once with their standing various paths.

\section{PROPOSED METHOLOGY}

\section{Independent Route Discovery in MANET}

In Mobile Ad hoc networks (MANET), the active node structure triggered by node movement which indicates an unfortunate route strength and consequently consumes a negative impression on network routines. In MANET the currentinclination in routing is to achieve routing information, once the routes are conventional only if it is essential. Several protocols in MANET are unicast like AODV and DSR, these protocols determination to never use multiple paths. Oncethe route disconnects then the nodes of the damaged route evenhandedly drop the data packets in an exact path to route the destination until a novel route is opportunity to deliver the data through route creation and consequently cannot be stable in delivering packets in different routes and that is a major problem identified in existing terminology. So it is focused to implement DSR protocol where it is used, when route is created between nodes and also it reduces overhead and collision.

In our proposed approach, frequently it is to find the sequence of neighbor nodes which possess a table of their furthermost recent node information with all other connected nodes in MANET.Asequential node come upon withtwo nodes transpiresonceindividualsource nodeexactly one-hop with neighbors.Subsequently one-hop neighbor node which has recent table with updated time stamp route map which is dependent on the link layer,the particularnode circumstance will come across to ensurethe condition depending up on sequential node search. It is to focus once a source node is ready to establish a connection to destination then the route calculation is adopted and it is essentialto examine the network until it discovers both thedestination andalternative node which takes a path to the destination. The proposed protocols for ad hoc networkswhich perform a recent route history based route discovery from neighboring node, whereby a recent route request (RRREQ) packet is gathered across the neighboring node in network, probablyconsumingvery low energy to update source own table which plays an major role in MANET environment.

Respective route deployment path way has been accomplished with the route discovery approach from source node as follows, the network, subsequently no replacementfor nodes to take recognized. Accordingly it ensures not todeliver the

1. Source node $\rightarrow$ Sequential node search $\rightarrow$ path discovery $\rightarrow$ recent time stamp $\rightarrow$

Connected node $\rightarrow$ Quick request responds (QRR)

2. Neighboring node $\rightarrow$ Updated table $\rightarrow$ recent route discovery $\rightarrow$ shortest path $\rightarrow$

Recent route request ( $R R R E Q$ )

3. Destination node $\rightarrow$ Frequent responds delivery $\rightarrow$ delay frequency $\rightarrow$

Delay ratio

4. Node life time $\rightarrow$ Enduring energy $\rightarrow$ past history $\rightarrow$ data transmission

Consumed $\rightarrow$ Energy exhaustion

Where destination node $\mathrm{D}$ findingsare basically from initialized source $S$ which activates the neighboring nodes formulated in equation (1.1) and (1.2) respectively.

Exploratory function $\mathrm{E}(\mathrm{x})$ followed by the neighboring node $\mathrm{d}(\mathrm{x}, \mathrm{y})$ denotes the length,

$$
E(x) \leq d(x, y)+E(y)
$$

From source $\mathrm{S}$ form an initial RRREQ to node $\mathrm{X}$, where length of the path denoted as $\mathrm{L}$ and $\mathrm{Y}$ is the routeX which encompasses to comprisey,

$$
L(X)+E(x) \leq L(X)+d(x, y)+E(y)=L(Y)+E(y)
$$

Similarly the length of the route $R_{l}$ represents route discovery with directly connected nodes and neighbor node $Z$ is for sequential search with route path $K$ from set of all nodes $\sum$ as shown in equation (2) respectively,

$$
R_{l}=\frac{\sum_{k=1}^{k=l-1} z_{l,} z_{n}\left|z_{l}-z_{l+1}\right|}{\left|z_{l}-z_{n}\right|}
$$

Responsibly it will make the connecting nodes more active with its responds towards route $R_{l}$ with respect to delay ratio.

\section{Route Discovery through Neighboring Node}

The estimated work towards route discovery from source node has been simplified with various neighboring resent route table history. In this approach destination node location and its distance can be calculated through latest and updated route table of neighboring node with respect to Quick Request Response (QRR) form requested nodes.

The source node has to find simplified and confirm shortest route to the destination formerlycommunicating any data packets through its connected link. This approach reflects with its time taken to enhance route discovery method through a nodes which is directly or jointly connected as a standard of measurement for route discovery practice. The route involves various associated nodes connected with each other to exchange route history specifically node which has latest route history and it will be identified through nodes time stamp (i.e.) latest packet exchange between nodes. In the beginning source node has been considered as $\mathrm{S}$, the destination node has been considered as D and all the other nodes are named in numerical numbers $(1,2 \ldots . .9)$.

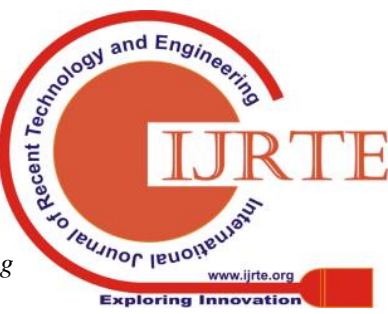


Firstly source node $S$ have sent route request (RREQ) to update their resent table history to the directly linked neighbor nodes (i.e.) namely $(S \rightarrow 1, S \rightarrow 3$ and $S \rightarrow 5)$ after the this request made to neighbor node, then the route $S \rightarrow 5$ has been identified as recently updated node to reach destination $\mathrm{D}$ from source $\mathrm{S}$ as shown in figure 1 .

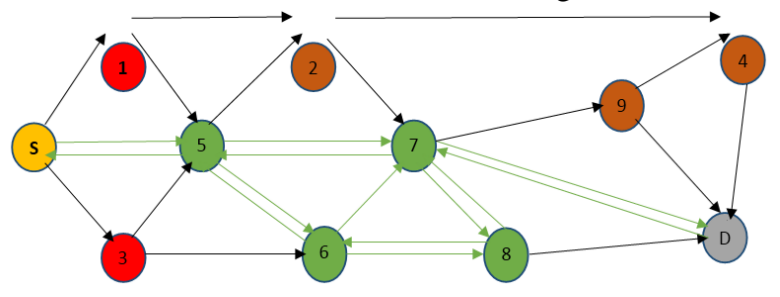

Figure 1: Route Discovery with Neighboring Nodes

Generally it is focused to set a frequent route discovery approach from source node which route are really essential to discover with extended route period with few changes in the MANET. Consequently the source can trigger the shortest path from the neighboring nodes table with its recent time stamp. Source node has a multipath way to reach the destination namely (P5, P7 and D) (P5, P6, P8 and D). If all the either paths fail then source jumps to the next route discovery process which is already found as shown in the table 1 and related sequence representation in figure 2 .

Table 1: QRR Route Discovery Path Sequence

\begin{tabular}{|l|l|l|l|}
\hline $\begin{array}{l}\text { Route } \\
\text { Discove } \\
\text { ry Node }\end{array}$ & $\begin{array}{l}\text { Path } \\
\text { Sequence }\end{array}$ & $\begin{array}{l}\text { Trigger } \\
\text { ed } \\
\text { Route }\end{array}$ & $\begin{array}{l}\text { Quick } \\
\text { Request } \\
\text { Responds(QR } \\
\text { R) }\end{array}$ \\
\hline $\mathrm{S}_{\mathrm{i}}$ & P1,P5,P3 & Nil & P5 \\
\hline $\mathrm{S} \rightarrow \mathrm{P} 1$ & Nil & Nil & Nil \\
\hline $\mathrm{S} \rightarrow \mathrm{P} 3$ & Nil & Nil & Nil \\
\hline $\mathrm{S} \rightarrow \mathrm{P} 5$ & $\begin{array}{l}\text { P5,P7and } \\
\text { D }\end{array}$ & P7 & P5 \\
\hline $\mathrm{S} \rightarrow \mathrm{P} 5$ & $\begin{array}{l}\text { P5,P6,P8 } \\
\text { and D }\end{array}$ & P6,P8 & P5 \\
\hline $\mathrm{D}_{\mathrm{j}}$ & $\begin{array}{l}\text { P4,P7,P8, } \\
\text { P9 }\end{array}$ & P7,P8 & P7 \\
\hline $\mathrm{S}_{\mathrm{i}} \rightarrow \mathrm{D}_{\mathrm{j}}$ & P5,P7 & P5,p7 & P5, P7 \\
\hline
\end{tabular}

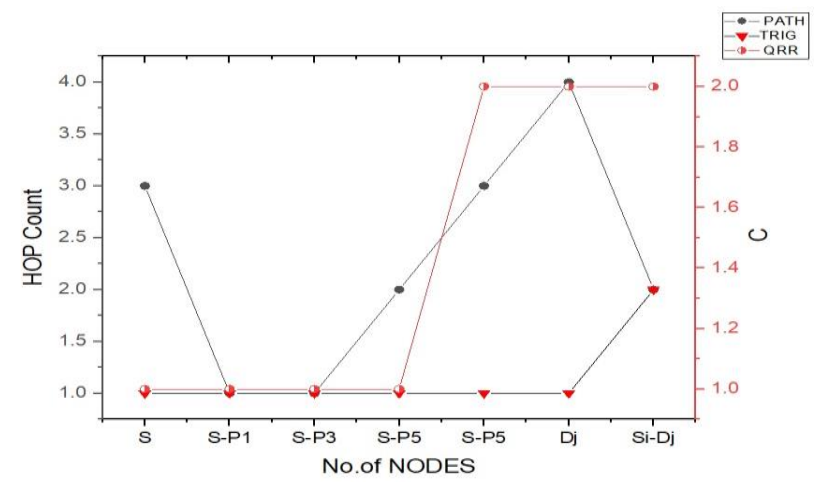

Figure 2: Triggered Route Path with QRR

When a source Node necessitateswith a route to a destination, a RREQnotethat encompasses the routing technique set is transmission. It is focused to build Proficient Route Encounter Algorithm (PREA) to redefine source node with recent node communication history from neighbor node as follows,

Simplified neighboring resent route table history with PREA: a) Initially unfilled routing path agreed with $(\mathrm{RPA}=$ $\varnothing)$.

b) First-rate up to three neighbors that have the latest node communication

History $S_{i}(1,5,3) \rightarrow$ source found node with recent communication.

c) Using the QRR from neighbor table, calculate shortest distance, triggered route

$\operatorname{Min}(S \rightarrow 5,7, D)$ and $\operatorname{Max}(S \rightarrow 5,6,8, D)$ in $R S$

Algorithm 1: PREA (neighbor's Resent Table):

- $\quad$ Initially $(\mathrm{RPA}=\varnothing)$.

- For each RPA node D do available max [hop]= count[3];

- For each RPA node D do available min [hop]= count[2];

- $\mathrm{RPA}=$ minimum ' $\mathrm{x}$ ' synchronized with the nodes arranged then change;

- $\quad$ Change =true;

- While change do begin

- $\quad$ Change $=$ false

- $\quad$ For each RPA node D do begin max[hop]

- $\operatorname{Max}[\mathrm{hop}]=$ maximum not predecessor path to D then exit;

- $\quad$ Old out $=\max$ out[D];

- $\quad$ Max out[D]= gen[D] Ù (in[D] - kill[D])

- If max out[D] "old out" then change = true;

- Create a new shortest path link

- $\quad$ End

\section{Avoiding End to End Delay Ratio between Nodes}

The route performance between nodes consumes a routing protocols exploration intended for paths with necessary updated routes to content the source nodeswhich desires a sequenceof flow. The updated route history and its routing protocol from neighboring nodes should had better path that connected with minimum network resources which can reduce the delay frequency between nodes. Similarly the solicitation elaborate MANET's limitations that might be accessible in network bandwidth, end-to-end delay between nodes, delay ratio, delay deviation, resource energy, possibility of data loss etc. The delay variation metrics between end nodes are categorized as improver metrics, energy metrics and multiple node metrics.

Consider the notation $\mathrm{m}(\mathrm{s}, \mathrm{d})$ be the routine metric for the association (s,d) joining node $\mathrm{s}$ to node $\mathrm{d}$, and path $(\mathrm{s} 1, \mathrm{~s} 2, \mathrm{~s} 3 \ldots . \mathrm{d})$ a classification of associations for the path from $\mathrm{s}$ to $\mathrm{d}$. A constraint is improver if $\mathrm{m}(\mathrm{s}, \mathrm{d})=\mathrm{m}(\mathrm{s}, \mathrm{s} 1)+\mathrm{m}(\mathrm{s} 1, \mathrm{~s} 2)+\mathrm{m}(\mathrm{s} 3+\mathrm{s} 4)+\mathrm{m}(\mathrm{sj}, \mathrm{d})$. For example, the end-to-end delay $(\mathrm{s}, \mathrm{d})$ is an improver constraint since it involvesthe $\left(\sum\right)$ of end to end delay for each associationlaterallywith the recent path.A constraint is energy metrics if $\mathrm{e}(\mathrm{s}, \mathrm{d})=\min \{\mathrm{m}(\mathrm{s}, \mathrm{s} 1), \mathrm{e}(\mathrm{s} 1, \mathrm{~s} 2), \ldots, \mathrm{e}(\mathrm{sj}, \mathrm{d})\}$. 
A constraint is multiple nodes if $\mathrm{m}(\mathrm{s}, \mathrm{d})=\mathrm{m}(\mathrm{s}, \mathrm{s} 1) \mathrm{x}$ $\mathrm{m}(\mathrm{s} 1, \mathrm{~s} 2) \times \ldots \times \mathrm{m}(\mathrm{sj}, \mathrm{d})$. The possibility of a packet delay ration lays on $\mathrm{p}(\mathrm{s}, \mathrm{d})$, directed from source node $\mathrm{s}$ to reach a destination node $d$ are always multiple in terms of between nodes as it is the product of distinct nodes possibilities of end to end route map. The trustworthiness of a connected nodes is based on improver metrics, energy ratio and delay ratio between source to destination increases or decreases with respect to multiple node metrics.

Initially source REQ to connected nodes are frequently monitored to build a link between nodes to destination based on delay ratio. Every node in MANET depends upon low delay ratio frequency for faster and smoother communication.

Here delay ratio has been calculated based on the table history of the link nodes with respect to multiple nodes between source and destination. An EDR (Estimated Delay Ratio) algorithm to find low delay nodes path and select theroot node to send and receive data packets as follows,

\section{Algorithm 2: Estimated Delay Ratio between nodes (EDR)}

- Read the number of node directly connected to source $S$

- Source REQ query to linked nodes assume x,y

- Check if linked node table $\geq$ table recent history if 'yes' move to step 4

Else 'Terminate'

- Check if estimated delay ratio $\leq$ Low if 'yes' move to step 5

Else 'Terminate'

- Check if delay ratio is acceptable and $\leq$ estimated then move to step 6

Else 'Terminate'

- If all step 3,4 and 5 satisfactory then forward REQ to next node

- If next node is destination then establish link between source to destination

Else go-back-to step 3

- $\quad$ Else send packets

- End if

- End if

- End

The above prompted EDR algorithm is to take certain route maintenance from source to destination which is specifically used to detect failure links in the path which is currently in use. In the other end source node unaware of link failure between nodes, whether the link to its downstream connecting node path is damaged.

Subsequently an intermediate node sends reverse notification or source can detect by monitoring delay ratio, which notifies source by sending a Route Error (RERR) dispatch to specify the node failure between nodes with Incremental Delay Frequency (IDF) as shown in equation (3) where $R_{f}$ as route failure with the sequence number of node $N_{(n-1)}$ with the maximum failure node $F_{(n-1)}$ with distance between nodes as $D_{(n-1)}$ with respect to route maintenance $R_{(m-l)}$ respectively,

$$
I D F=\frac{R F\left[N_{(n-1)}+F_{(n-1)}+D_{(n-1)}\right]}{R_{(m-1)}}
$$

Accordingly the source node $S_{i}$ triggered the path with minimum delay ratio with the destination $D_{j}$. End to End delay is frequently updated with neighboring nodes estimated delay frequency with respect to a recent history as shown in table 2 and figure 3.

Table 2: End to End Delay Discovery

\begin{tabular}{|l|l|l|l|}
\hline $\begin{array}{l}\text { Route } \\
\text { Discovery Node }\end{array}$ & $\begin{array}{l}\text { End To } \\
\text { End Delay }\end{array}$ & $\begin{array}{l}\text { Estimated } \\
\text { Delay }\end{array}$ & $\begin{array}{l}\text { Delay } \\
\text { Ratio }\end{array}$ \\
\hline $\mathrm{S}_{\mathrm{i}}$ & 0.1 & 0.2 & 0.1 \\
\hline $\mathrm{S} \rightarrow \mathrm{P} 1$ & 1 & 1.2 & 0.2 \\
\hline $\mathrm{S} \rightarrow \mathrm{P} 3$ & 2.2 & 2.5 & 0.3 \\
\hline $\mathrm{S} \rightarrow \mathrm{P} 5$ & 1.8 & 2 & 0.2 \\
\hline $\mathrm{D}_{\mathrm{j}}$ & 0.1 & 0.2 & 0.1 \\
\hline $\mathrm{S}_{\mathrm{i}} \rightarrow \mathrm{D}_{\mathrm{j}}$ & 3 & 3.2 & 0.2 \\
\hline
\end{tabular}

After such scenarios the proposed approach focused with the source node exit from the present connected route and the specious hop from its updated nodes table by paring all routes that contain this sequential hop, consumptions additional table route to initiate packets transmission between nodes. Uncertainly there is no alternate route with recent history is available then source node $S_{i}$ accomplishes route encounter over to obtain a novel and shortest route with minimum delay to the destination $D_{j}$ with highest packet delivery as shown graphical view in figure 3 .

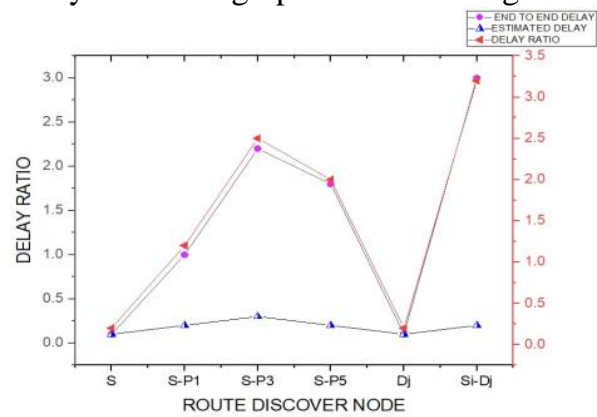

Figure 3: End to End Route Discovery

\section{RESULTS AND DISCUSSION}

It is focused to implement our algorithm and protocol used in Network Simulator which is commonlyrecognized as NS2 itis basically an experiencedetermined simulation tool. A simulation study was carried out to calculate the performance measurements of MANET routing protocols such as DSR with proposed algorithm PREA and EDRestablished on the end-to-end delay, throughput and packet delivery ratio average with the subsequent parameters

\section{Simulation Parameters}

\begin{tabular}{|l|l|}
\hline Parameter & Value \\
\hline Transmission range & $150 \mathrm{~m}$ \\
\hline Protocols \& Algorithm & DSR, PREA and EDR \\
\hline Traffic Source & Constant Bit Rate \\
\hline Packet size & 512 bytes \\
\hline Data rate & $128 \mathrm{kbps}$ \\
\hline Area & $500 \times 500$ \\
\hline Number of nodes & 100 \\
\hline Application & FTP \\
\hline MAC & IEEE 802.11 \\
\hline Simulation time $(\mathrm{Sec})$ & $10,20,30,40,50,60,70,80,90 \& 100$ \\
\hline
\end{tabular}

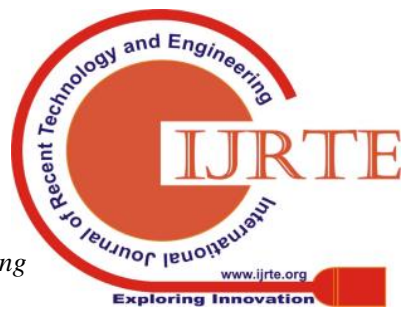




\section{End to End Delay}

In the proposed simulation environment the packet end to end delay is the typical time that a packet proceeds to navigate the network. The time taken from the source which generates the packet active to the situation response at the destination where it is restrained in seconds. It is initiated to use 15 to 100 nodes with maximum time value of 100 seconds. It consequently contains all the delays in the network such communication time and delay between nodes which prompted by routing events and MAC regulator interactions. Therefore end to end delay ratio is improvised as shown in equation 4, where ATP (Arrival Time of Packet) and STP (Send Time of Packet), it is measured with the routing protocol and algorithm driven in table 3 and figure 4 .

$$
\text { End to End delay }=\sum \frac{A T P-S T P}{\text { No.ofconnections }}
$$

Table 3: End to End Delay Ratio

\begin{tabular}{|l|l|l|l|l|}
\hline \multicolumn{5}{|l|}{ End to end delay ratio } \\
\hline No. of nodes & Time/ Sec & DSR & PREA & EDA \\
\hline 15 & 10 & 0.01248 & 0.151 & 0.142 \\
\hline 30 & 25 & 0.11337 & 0.305 & 0.299 \\
\hline 45 & 40 & 0.21265 & 0.452 & 0.401 \\
\hline 60 & 55 & 0.41374 & 0.657 & 0.599 \\
\hline 75 & 70 & 0.61249 & 0.752 & 0.740 \\
\hline 90 & 85 & 0.81648 & 0.901 & 0.902 \\
\hline 100 & 100 & 1.01238 & 1.004 & 1.003 \\
\hline
\end{tabular}

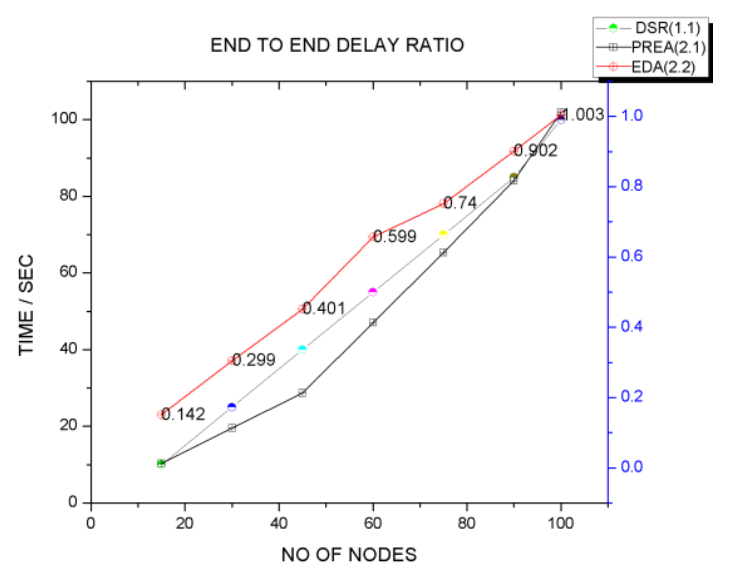

Figure 4.1: End to End Delay Ratio with DSR, PREA and EDA

It is executed to authenticate the performance analysis of data rate of packet sent from source to destination. Frequently it is analyzed to retrieve throughput for future path connectivity. The totalsize of data ratio that ranges since source to the period it receipts for the destination to get the previous packet. After associating the routing throughput by theprotocols DSR with algorithm driven has the high throughput comparatively. The throughput rate of DSR gradually grows primarily andupholds its significance when the time increasesas shown intable 5.Finally throughput has Total Packet Received (TPR), Packet Size (PS) and Simulation Time (ST) it was analyzed in the

\section{Throughput}

following equation 5 with maximum time sequence in table 4 and figure 5 .

$$
\text { Throughput }=\frac{T P R^{*} P S}{S T}
$$

Table 4: Throughput

\begin{tabular}{|l|l|l|l|l|}
\hline \multicolumn{5}{|l|}{ Throughput } \\
\hline No. of nodes & Time/ Sec & DSR & PREA & EDA \\
\hline 15 & 10 & 0.31248 & 0.451 & 0.342 \\
\hline 30 & 25 & 0.41337 & 0.505 & 0.299 \\
\hline 45 & 40 & 0.51265 & 0.452 & 0.401 \\
\hline 60 & 55 & 0.61374 & 0.657 & 0.699 \\
\hline 75 & 70 & 0.61249 & 0.752 & 0.840 \\
\hline 90 & 85 & 0.81648 & 0.901 & 0.902 \\
\hline 100 & 100 & 1.91238 & 1.302 & 1.401 \\
\hline
\end{tabular}

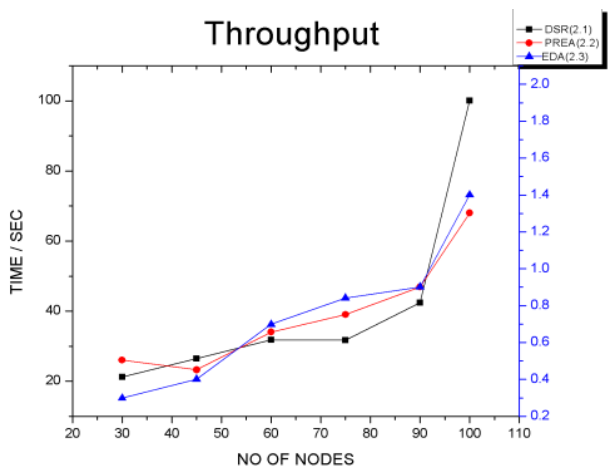

Figure 4.2: Throughput with DSR, PREA and EDA

\section{Packet Delivery Ratio}

It is most important scenario to validate the Packet Delivery Ratio (PDR) between the source and destination with their sequential path nodes. The number of packets generated from source and it is transmitted by a trafficsource and the number of packets received by a receiver end traffic as logged in the trace file. It measures ratio of packet delivery in between the received packets in the destination end and generated packets from the source end. The loss rate has been sequentially analyzed from transport protocols and it will be describedwith both accuracy and effectiveness of DSR routing protocol. An extraordinary packet delivery ratio is anticipated with the driven algorithm both PREA and EDA with the following equation 6 as Number of Packet Received (NPR), Number of Packet Sent (NPS) and its respective values in table 5 and figure 6 .

$$
\mathrm{PDR}=\sum \frac{N P R}{N P S}
$$

Table 5: Packet Delivery Ratio

\begin{tabular}{|l|l|l|l|l|}
\hline \multicolumn{5}{|l|}{ Packet delivery ratio } \\
\hline No. of nodes & Time/Sec & DSR & PREA & EDA \\
\hline 15 & 10 & 0.61748 & 0.551 & 0.445 \\
\hline 30 & 25 & 0.71837 & 0.599 & 0.699 \\
\hline 45 & 40 & 0.81965 & 0.652 & 0.678 \\
\hline 60 & 55 & 0.81774 & 0.699 & 0.799 \\
\hline 75 & 70 & 0.91949 & 0.878 & 0.885 \\
\hline 90 & 85 & 0.91848 & 0.988 & 0.965 \\
\hline 100 & 100 & 1.91938 & 1.504 & 1.606 \\
\hline
\end{tabular}




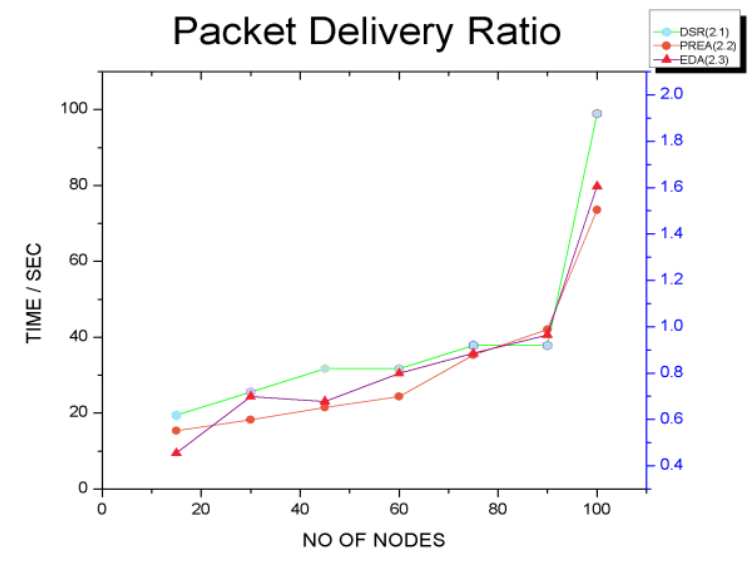

Figure 4.3: Packet Delivery Ratio with DSR, PREA, EDA

\section{CONCLUSION}

In this research paper the significant idea is to direct the route path request from source and update its destination route map with its neighboring nodes recent table history. This sufficient pupation will make source node more active in finding destination route path with minimum time conception. The impartial of the work is to minimize the overhead parameter such as End-to-End delay, throughput, and packet delivery ratio over by varying network size simulationtime comparing with existing method.The proposed method be able to use in avoiding maximum time taken to find the exact route path for data delivery. By comparing with the existing methodit is focused to permit better and effective use of proposed algorithm PREA and EDA for updating recent history from neighboring nodes and it will make sure of terminating nodes route path with old history which will be more suitable for reducingrouting traffic due to route discovery. The QRRfrom neighbor node using an existing updated route will bring the route demand closer to thedestination with the support of location information. The performance has been analyzed and verified with MANET Routing protocol DSR with two proposed algorithm PREA and EDA using NS-2 Simulator to achieve better and resourceful route discovery.

\section{REFERENCES}

1. R. Rajeshkanna, S. Poorana Senthilkumar, C. Kumuthini, "Energy Efficient Routing Protocols in Mobile Ad hoc Network Based on Enhanced AODV Protocol", IJCSMC, vol.2, issue.7, pp.391-395, 2013..

2. S.-J. Lee, E. M. Belding-Royer, C. E. Perkins, Scalability study of the ad hoc on-demand distance vector routing protocol, Int. J. Netw. Manag. 13 (2003) 97-114.

3. K. Du, Y. Yang, A qos routing for maximum bandwidth in ad hoc networks, in: ICFN '10: Proceedings of the 2010 Second International Conference on Future Networks, IEEE Computer Society, Washington, DC, USA, 2010, pp. 343-345. Y. H. Ho, A. H. Ho, K. A. Hua, Routing protocols for inter-vehicular networks: A comparative study in high-mobility and large obstacles environments, Comput. Commun. 31 (2008) 2767-2780. URL http://dl.acm.org/citation.cfm?id=1389585.1389891

4. J. Ichikawa, S. Sakata, and N. Komuro: "Fair Energy Consumption- Based Routing Control Considering Data Trafic for Mobile Ad hoc Networks," IEICE Technical Report, IN2013-171, pp.163-168 (2013) (In Japanese).
5. Henri Dubois Ferriere Matthias Grossglauser Martin Vetterli School of Computer and Communication Sciences EPFL 1015 Lausanne, Switzerland Age Matters: "Ef£cient Route Discovery in Mobile Ad Hoc Networks Using Encounter Ages" MobiHoc'03, June 13, 2003, Annapolis, Maryland, USA. Copyright 2003 ACM 1581136846/ 03/0006

6. X. Wu, J. Wang, and C. Wang, "Stability-enhanced routing for Mobile ad hoc Networks," presented at International Conference on Computer Design and Applications (ICCDA 2010), 2010.

7. W. Su, S. J. Lee, and M. Gerla, "Mobility prediction and routing in ad hoc wireless networks," Int. J. Netw. Manage. IEEE transaction vol. 11, no. 1, pp. 3-30, 2011.

8. R. Dube, C. D. Rais, K. Y. Wang, and S. K. Tipathi, "Signal stability based adaptive routing (SSA) for ad hoc mobile networks," IEEE Papers Commun, vol. 4, no. 1, pp. 598-598, 2010.

9. R. Dube, C. D. Rais, K. Y. Wang, and S. K. Tipathi, "Signal stability based adaptive routing (SSA) for ad hoc mobile networks," IEEE Papers Commun, vol. 4, no. 1, pp. 598-598, 2012.

10. M. Yoshimachi and Y. Manabe: "Battery Power Management Routing Considering Participation Duration for Mobile Ad HocNetworks," JACN 2016 Vol.4, pp. 1318. (2015).

11. Harmanpreet Kaur, Puneet Kumar, "Time base control model for efficient transmission in MANET", vol.4, issue.3, pp. 166-168, 2016.

12. Anumeha, Prof. (Dr.) Bhawna Mallick, "Introducing efficient \& improved. AODV routing protocol for MANET”, IJSTM, vol-no.4, pp.36-44, 2015.

13. Kanojia Sindhuben Babulal and Rajiv Ranjan Tewari, E2XLRADR (Energy Efficient Cross Layer Routing Algorithm with Dynamic Retransmission for Wireless Sensor Networks), International Journal of Wireless \& Mobile Network, Vol. 2, No. 3, pp. 167-177, Aug. 2010. 